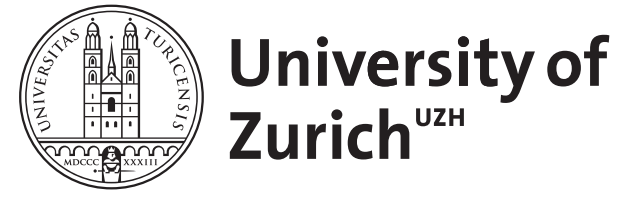
Archive

University of Zurich

University Library

Strickhofstrasse 39

CH-8057 Zurich

www.zora.uzh.ch

Year: 1988

\title{
More on an Easily Obtained Accurate Dialysis Index
}

Probst, W ; Binswanger, U

DOI: https://doi.org/10.1093/oxfordjournals.ndt.a091736

Posted at the Zurich Open Repository and Archive, University of Zurich

ZORA URL: https://doi.org/10.5167/uzh-155649

Journal Article

Published Version

Originally published at:

Probst, W; Binswanger, U (1988). More on an Easily Obtained Accurate Dialysis Index. Nephrology, Dialysis, Transplantation, 3(5):706-707.

DOI: https://doi.org/10.1093/oxfordjournals.ndt.a091736 
A 58-year-oid Caucasian man was admitted for evaluation: of a nephrotic syndrome. He had a past history of mild hypertension which had been treated with a combination of hydrochlorothiazide and amiloride. Blood pressure was $130 / 70 \mathrm{mmHg}$; there was no oedema. Serum electrolytes were normal, creatinine $112 \mu \mathrm{mol} / \mathrm{l}$, albumin $29 \mathrm{~g} / \mathrm{l}$, gammaglobulin $5.3 \mathrm{~g} / \mathrm{l}$, total cholesterol $10.2 \mathrm{mmol} / \mathrm{l} ; 24 \mathrm{~h}$ urinary protein ranged from 3 to $5 \mathrm{~g}$; urinary sediment was normal, and urine culture sterile. GFR was $86 \mathrm{ml} / \mathrm{min}$ per $1.73 \mathrm{~m}^{2}$, as assesssed by ${ }^{129}$ I Iothalamate clearance. Light microscopy and immunofluorescence studies of the specimens obtained by percutaneous renal biopsy revealed a typical pattern of stage 2 membranous nephropathy.

Further evaluation showed normal blood cell count, serum complement, alphafetoprotein, and carcinoembryonic antigen; there was absence of anti-DNA antibodies, hepatitis B virus markers, antithyroglobulin and antimicrosomal antibodies, cryoglobulinaemia, and mercury in urine. VDRL and FTA tests were negative. There was no evidence of neoplasia, sarcoidosis, or multisystemic or infectious diseases.

Drug inquiry revealed that the patient had been taking fenoprofen $600-1500 \mathrm{mg}$ daily for the preceding 12 months, because of loin pain following a vertebral trauma. Fenoprofen was with. drawn while antihypertensive treatment was maintained, and complete remission of proteinuria occurred within 6 months, without steroid or immunosuppressive therapy, and was persisting 2 years later.

Nephrotic syndrome induced by NSAID has been related to minimal-change glomerulopathy and has been most often associated with acure renal failure and severe intersitial nephritis [1]. NSAID-associated membranous glomerulonephritis is extremely unusual. We are aware of only four reported cases, three with diclofenac [2-4] and one with ketoprofen, a propionic-acid derivative like fenoprofen [5]. All patients presented with the nephrotic syndrome, and complete remission occurred in all within 12 months of stopping the presumed causative drug.

Thus, a search for a prior NSAID treatment should be included in the drug inquiry conducted in every patient with membranous glomerulonephritis.
Division of Nephrology

Lyon-South University Medical Centre

France
69310 Pierre-Bẻnite
R. Cahen

P. Trolliet

B. Francçois

C. Chazot
1. Clive DM, Stoff JS. Renal syndromes associated with nonsteroidal anti-inflammatory drugs $N$ Engl J Med 1984: 310: 563-572

2. Ducret F, Pointet P, Pichot C. Membranous glomerulonephritis during rheumatoid arthritis. Probable toxicity of diclofenac. Nephrologie 1980; 1: 143-144

3. Hurault de Ligny B. Faure G. Bene MC, Kessler M, Huriet C. Extra-membranous glomerulonephritis during the treatment of rheumatoid polyarthritis with diclofenac. Nephrologie 1984; 5 : $135-136$

4. Schillinger F, Montagnac R. Milcent T. Membranous glomerulonephritis after diclofenac treatment. Kidney Int 1987; 32: 428-429, (Abstract)

5. Sennesaeal J. Van Den Houtte K. Verbeelen D. Reversible membranous glomerulonephritis associated with ketoprofen. Clin Nephrol 1986: 26: 213-215

\section{Systemic and Disseminated Candidiasis Complicating Renal Failure}

Sir,- -

We read with great interest the paper 'Systemic and disseminated candidiasis complicating acute renal failure' by Stevens and co-workers [1].
It was rather disconcerting to note that none of their patients were on prophylactic oral antifungal treatment. In a controlled trial Ledingham and co-workers [2] using a Selective Parenteral and Enteral Antisepsis Regimen (SPEAR) which included systemic cefotaxime, oral polymyxin $E$, tobramycin and amphotericin $B$ were able to demonstrate a substantial reduction in the incidence of acquired infection ( $10 \%$ vs $24 \%$ ). The isolation of yeasts from stomach, throat and trachea declined appreciably during days $2-4$ in the test group, but changed little with time in the control group.

It has been our time-honoured practice to give nystatin 100000 units 6-hourly to all patients admitted to our Regional Renal Unit, irrespective of the type of renal failure, thereby obtaining gratifying results. However, we were recently involved in the management of a patient with acute myeloid leukaemia who had not received oral nystatin and had developed hepatic candidial abscesses and septicaemia; despite treatment with amphotericin $B$ and flucytosine, the patient died with renal failure.

One of us (P.McC) recently investigated 27 patients who required ventilation and dialysis for a period of more than 5 days. Cultures (1641) were taken from tracheal aspirate, urine, wound and blood. Of 1011 cultures in 15 patients using SPEAR therapy, 103 were positive for yeasts $(10.4 \%)$. Of the $630 \mathrm{cul}$ tures taken in the remaining 12 control patients, 137 were positive for yeasts $(21.7 \%)$. Our study is in agreement with the findings reported by Ledingham and co-workers [2]. We cannot but emphasise the importance of oral antifungal therapy, which is not only a simple, but also a cheap method of preventing candidiasis.

Regional Renal Unit

Royal Liverpool Hospital

M. Yaqoob

Prescott Street

P. McClelland

Liverpool, UK

R. Ahmad

1. Stevens PE, Rainford DJ, Green AD, Masterton RG. Systemic and disseminated candidiasis complicating acute renal failure. Nephrol Dial Transplant 1988; 3: 84-86

2. Ledingham IA, Eastaway AT, McKay IC, Alcock SR, McDonald JC, Ramsay G. Triple regimen of selective decontamination of digestive tract, systemic cefotaxime, and microbiological surveillance for prevention of acquired infection in intensive care. Lancet 1988; 1 : 785-790

\section{More on an Easily Obtained Accurate Dialysis Index}

Sir,-

The search for quantification of dialysis treatment as stimulated by the NCDS study resulted in different approaches to collecting data in order to calculate $\mathrm{K} \times \mathrm{t} / \mathrm{V}(\mathrm{K}=$ clearance; $\mathrm{t}=$ treatment time; $V=$ distribution volume). $K$ was most often taken from in vitro data provided by the manufacturers of filters, whereas the distribution volume was either set at $0.58 \times$ bodyweight, or was calculated from urea extraction divided by change of blood urea concentration before and after treatment.

We measured urea extraction by collecting dialysate, and obtained measurements for blood urea before and after treatment. Results for $\mathrm{K} \times \mathrm{t} / \mathrm{V}$ obtained from these 'true data' were compared with those obtained from both calculated and in vitro data. Furthermore, a formula is derived which permits the estimation of the 'true' dialysis index from blood urea measurements before and after treatment. 


\begin{tabular}{llccl}
\hline Calculation mode & $K$ & $V$ & $K \times 1 / V$ \\
\hline & $K:$ in vitro data & $225.36 \pm$ & $34.34 \pm$ & $1.05 \pm$ \\
V: $0.58 \times \mathrm{BW}$ & 25.15 & 4.56 & 0.15 \\
& K:in vivo data & $173.00 \pm$ & $34.34 \pm$ & $0.80 \pm$ \\
& V: $0.58 \times \mathrm{BW}$ & $23.69^{*}$ & 4.56 & $0.09^{*}$ \\
$\mathrm{~K}:$ in vivo dialysate & $173.00 \pm$ & $39.12 \pm$ & $0.74 \pm$ \\
& V: and blood urea & $23.96^{*}$ & 10.95 & $0.17^{*}$ \\
\hline
\end{tabular}

$\mathrm{M} \pm \mathrm{SD} ; n=14 ;{ }^{*}$ Significance: 1 vs 2,1 vs $3: P<0.001$

Results (Table) indicate that the 'true' dialysis index accord. ing to formula $\mathrm{F} 3$ is $25 \%$ lower than the calculated value using in vitro clearance data and $0.58 \times \mathrm{BW}$ distribution volume (F1). Replacing the estimated clearance data by measured values (F2) adapts the index value partially to the true finding. The calculation of the 'true' dialysis index from extracted urea in dialysate and concentration pre- and post-treatment included

$$
\text { clearance }(K)=\frac{\text { urea extracted } / \text { treatment time }}{\text { (conc. pre }+ \text { conc. post }) / 2}
$$

urea distribution volume $(\mathrm{V})=\frac{\text { urea extracted }}{\text { conc. pre }- \text { conc. post }}$

Combining these formulae for calculation of

$$
\begin{gathered}
I=\frac{K \times t}{V} \text { results in } \\
I=\frac{\text { conc. pre }- \text { conc. post }}{(\text { conc. } p r e+\text { conc. post }) / 2}
\end{gathered}
$$

In conclusion, the 'true' dialysis index, taking into account in vivo filter clearance and individually calculated distribution volume, is easily obtained from routine pre- and post-dialysis blood urea measurements.

Note added in proof: This formula was recently described by Barth and Berlyne in abstract form (Kidney International 1988; $33,216)$

Section of Nephrology

Department of Internal Medicine

University Hospital Zürich, Switzerland

\section{Treatment of Pseudomonas aeruginosa Peritonitis in a CAPD Patient with Ciprofloxacin}

\section{Sir,-}

CAPD-associated peritonitis caused by $P$ seudomonas aeruginosa is a very serious complication of CAPD treatment, often resulting in catheter removal. Ciprofloxacin, one of the new quinolones, seems to have promising properties for the treatment of serious Pseudomonas aeruginosa infections [1,2].

A 58-year-old woman treated by CAPD for 10 months suffered five episodes of Staphylococcus aureus peritonitis. She developed a further episode of peritonitis and Pseudomonas aeruginosa was cultured from the effluent fluid. She was treated with intraperitoneal gentamicin and ticarcillin for 8 days and after 4 days of treatment the cultures became negative. Therapy was continued, but after another 4 days $P$ seudomonas aeruginosa was again cultured.

Ciprofloxacin was started with an initial intravenous dose of $400 \mathrm{mg}$, followed by intraperitoneal administration of $50 \mathrm{mg}$ q.d.s. Serum levels were between 0.2 and $0.6 \mathrm{mg} / \mathrm{l}$ and peritoneal dialysate levels varied between 1.4 and $2.2 \mathrm{mg} / \mathrm{t}$. The minimum inhibitory concentration (MIC) of ciprofloxacin for the Pseudomonas aeruginosa strain was $0.05 \mathrm{mg} / \mathrm{l}$. After 2 days, dialysate cultures became negative. Because of persistent high leucocyte counts in the peritoneal fluid, gentamicin was added to the regimen after 8 days of ciprofloxacin therapy. The cultures remained negative and leucocyte counts returned to normal after another 3 days. No side-effects were observed.

Ciprofloxacin has a bactericidal action against Gramnegative bacteria including Pseudomonas aeruginosa [3]. The quinolones have a low incidence of side-effects [4]. Ciprofloxacin was very well tolerated by our patient. Intraperitoneal doses of $50 \mathrm{mg}$ q.d.s. resulted in adequate ciprofloxacin levels in the peritoneal dialysate, which were 30 to 40 times the MIC value of the infecting organism. Ciprofloxacin seems to be a very promising drug in the treatment of CAPD-associated peritonitis caused by Pseudomonas aeruginosa.

Departments of Medicine and

Medical Microbiology

W. Grave

St Laurentius Hospital

Post Box 920, 6040 AX Roermond

The Netherlands

1. Bayer AS, Blomquist IK, Kwang Sik Kim. Ciprofloxacin in experimental aortic valve endocarditis due to Pseudomonas aeruginosa. $J$. Antimicrob Chemother 1986; 17:641-649

2. Isaacs D, Slack MPE, Wilkinson AR, Westwood AW. Successful treatment of pseudomonas ventriculitis with ciprofloxacin. $J$ Antimicrob Chemother 1986; 17: 535-538

3. Jones BM, Geary I, Lee ME, Duerden BI. Activity of pefloxacin and thirteen other antimicrobial agents in-vitro against isolates from hospital and genitourinary infections. J Antimicrob Chemother 1986; 17 : 739-746

4. Scully BE, Neu HC, Parry MF, Mandell W. Oral ciprofloxacin therapy of infections due to Pseudomonas aeruginosa. Lancet 1986; I: 819-822

\section{A Modification to the 'Shaldon Technique' when Inguinal Fibrosis is Present}

\section{Sir,-}

Shaldon's introduction of the Seldinger technique to catheterise the femoral vein [1] was a milestone in the management of the uraemic patient. Since then thousands of patients with acute renal failure and chronic renal insufficiency have benefited from this procedure. However, its repeated use has occasionally resulted in a zone of fibrous tissue in the inguinal region due to either consecutive localised haematomas and/or the effect of the local anaesthesia. In these cases its use is then not viable, as even if the vessel can be localised and the guidewire inserted, the catheter cannot be advanced because of the underlying fibrosis.

Although the development of different vascular access techniques [2], the use of unipuncture systems for undertaking haemodialysis sessions [3], or the permanence of catheters in the femoral vein for long periods of time [4] have decreased the incidence of this problem, it is still necessary on occasions to resort to the use of the classic Shaldon technique for the introduction of a vascular catheter.

We suggest a modification in the technique described by Shaldon to introduce a femoral venous catheter through an inguinal region when fibrosis makes its use impracticable. The femoral vein is localised in the traditional way, using a needle of Cournand through which a guidewire is passed. The needle is 